\title{
Les cellules souches mésenchymateuses favorisent la cicatrisation des lésions cutanées radio induites
}

\author{
S. FRANÇOIS ${ }^{1}$, M. MOUISEDDINE ${ }^{1}$, N. MATHIEU ${ }^{1}$, A. SEMONT ${ }^{1}$, P. MONTI $^{1}$,

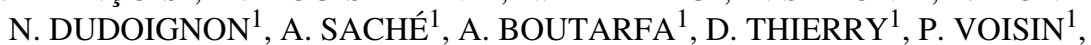 \\ P. GOURMELON ${ }^{1}$, A. CHAPEL ${ }^{1}$
}

(Manuscrit reçu le 6 juillet 2006, accepté le 16 septembre 2006)

RÉSUMÉ De nombreuses études suggèrent que les cellules souches adultes et plus particulièrement les cellules souches Mésenchymateuses humaines (CSMh) pourraient être utilisées pour réparer de nombreux organes. Nous avons étudié la capacité des CSMh à réduire les lésions cutanées radio induite. Pour induire des lésions sévères de la peau, des souris NOD/SCID ont été irradiées au niveau de la patte droite postérieure (30 Gy, débit $2,7 \mathrm{~Gy} / \mathrm{mn}$ ) en utilisant une source gamma au ${ }^{60}$ Co. Les CSMh ont été injectées 24 heures après irradiation par voie intraveineuse. La présence de cellules humaines, la sévérité des lésions et les processus de cicatrisation ont été étudiés sur les échantillons de peau prélevés de 3 à 8 semaines après irradiation. Nous avons pu observer que chez les souris greffées avec des CSMh, le niveau d'atteinte cutanée radio induite est significativement plus faible. Les scores cliniques utilisés pour l'étude de l'évolution des lésions cutanées de la peau sont significativement améliorés et une cicatrisation plus rapide est observée en comparaison des souris non injectées. La présence de cellules humaines a pu être détectée par PCR quantitative dans les zones cutanées en cours de cicatrisation. Ces résultats suggèrent premièrement que les CSMh sont capables de coloniser la peau altérée par les rayonnements ionisants et deuxièmement qu'elles accélèrent le processus de réparation de ce tissu limitant ainsi les complications tissulaires radio induites. La greffe de CSMh pourrait être un traitement thérapeutique efficace des phases précoces du syndrome cutané radio induit

ABSTRACT Mesenchymal stem cells favour healing of the cutaneous radiation syndrome.

It has been suggested that human Mesenchymal Stem Cells (hMSC) could be used to repair numerous injured tissues. We have studied the potential use of hMSC in order to limit radiation-induced skin lesions. Immunodeficient NOD/SCID mice were locally irradiated to the leg $(30 \mathrm{~Gy}$, dose rate $2.7 \mathrm{~Gy} / \mathrm{mn})$ using a ${ }^{60} \mathrm{Co}$ source in order to induce a severe skin lesion. Cultured bone marrow hMSC were delivered intravenously to the mice. The irradiated skin samples were studied for the presence of the human cells, the severity of the lesions and the healing process. Macroscopic analysis and histology results showed that the lesions were evolving to a less severe degree of radiation dermatitis following hMSC transplant when compared to irradiated non-transplanted controls. Clinical scores for the studied skin parameters of treated mice were significantly improved. A faster healing was observed when compared to untreated mouse. Immunohistology and Polymerase Chain reaction (PCR) analysis provided evidence that the human cells were found in the irradiated

\footnotetext{
${ }^{1}$ IRSN, Direction de radioprotection de l'Homme, B.P. 17, 92262 Fontenay-aux-Roses Cedex, France.
} 
area. These results suggest a possible use of hMSC for the treatment of the early phase of the cutaneous radiation syndrome. A successful transplant of stem cells and subsequent reduction in radiation-induced complication may open the road to completely new strategies in cutaneous radiation syndrome therapy

Keywords: mesenchymal stem cells / healing / skin

\section{Introduction}

Parmi les accidents d'irradiation, $54 \%$ sont des irradiations externes et localisées (Lefaix et Daburon, 1998), elles conduisent dans bien des cas à une irradiation sévère du tissu cutanée entraînant chez les sujets irradiés un syndrome cutanée radio induit constitué de lésions précoces et tardives. Le syndrome cutané est également l'un des effets secondaires en radiothérapie et en radiooncologie. Chez des patients subissant des radiothérapies, $95 \%$ développent des réactions cutanées précoces ou tardives d'intensité variée. Le syndrome cutané d'irradiation dépend de la dose délivrée, du volume de tissu irradié, de la radiosensibilité propre aux patients et du délai écoulé après exposition. Son expression clinique évolue du simple érythème (coup de soleil) à l'ulcération des tissus irradiés (Archambeau et al., 1995). Classiquement, il est admis qu'une dose supérieure à 25 Gy est à l'origine d'une nécrose sans guérison se développant en quelques mois. Ces lésions cutanées se définissent comme un processus de réparation tissulaire pathologique et dépendent de la combinaison dans le temps des effets et de la réponse de l'épiderme et du derme sous-jacent (mésenchyme et vascularisation). L'état du support sur lequel repose le tissu cutané (muscle et vascularisation) conditionne également l'évolution du syndrome cutané d'irradiation (Hopewell, 1990 ; Peter, 2002, 2005). À l'heure actuelle, il n'existe pas de stratégie thérapeutique clairement définie pour traiter une personne irradiée accidentellement.

La thérapie cellulaire au service de la médecine régénératrice pourrait être une alternative dans le traitement des lésions radio induites. Elle est aujourd'hui largement utilisée dans le domaine de l'hématologie (greffe de cellules souches hématopoïétiques), des brûlures (greffe de peau) et elle en est à un stade plus expérimental pour les maladies hépatiques, le diabète et les affections neurodégénératives. Les connaissances récentes sur les cellules souches adultes et plus particulièrement sur les cellules souches mésenchymateuses (CSM) permettent d'envisager des applications de la thérapie cellulaire pour la cicatrisation cutanée. Utilisées en recherche, les CSM proviennent d'échantillons de moelle osseuse obtenus par ponction sternale ou de la crête iliaque. Chez l'homme, la thérapie cellulaire par les CSM (auto et allogreffe) a déjà été réalisée, mettant en évidence à la fois une absence de toxicité liée à leur injection (Koc et Lazarus, 2002) et leurs effets bénéfiques thérapeutiques dans certaines pathologies telles que l'ostéogenèse imparfaite (Le Blanc et al., 2005) ou la GVH (Graft Versus Host Disease) (Koc 
et Lazarus, 2001). Les CSM peuvent in vitro se différencier en de nombreux types cellulaires du mésoderme, de l'endoderme et de l'ectoderme et ont la capacité de migrer vers un organe lésé (Chapel et al., 2003 ; François et al., 2006).

En effet, nous avons précédemment mis en évidence que les CSM de primates non humains (Chapel et al., 2003) ainsi que les CSM humaines (François et al., 2006) avaient la capacité de migrer vers la peau à la suite d'une exposition aux rayonnements ionisants. Il a également été montré que l'altération tissulaire favorise la migration des cellules médullaires vers la peau et que ces dernières pouvaient se différencier en différents types cellulaires structurant la peau avec des morphologies identiques aux cellules résidentes (Badavia et al, 2003). La greffe de cellules médullaires chez la souris nude en présence de cellules issues du derme d'embryon de souris a permis de montrer que les cellules médullaires ont la capacité d'acquérir le phénotype de plusieurs types cellulaires appartenant à la peau (Kataoka et al., 2003). En cas d'altération tissulaire, la colonisation par les cellules médullaires augmente, dans la zone lésée (Borue et al., 2004). Ces cellules médullaires pourraient favoriser la cicatrisation par leur sécrétion de collagène (Fathke et al., 2004) et elles pourraient être impliquées dans la régénération de la peau (Brittan et al., 2005 ; Yamaguchi et al., 2005 ; Mori et al., 2005). Après injection intraveineuse de CSM issues de souris BalbC (pelage blanc) à des souris C57Black (pelage noir), l'apparition de poils blancs a permis de démontrer que les CSM sont capables de se substituer aux cellules souches de la peau (Weimin et al., 2005). Les CSM humaines semblent pouvoir contribuer à la régénération de la peau chez la souris (Mansilla et al., 2005, Nakagawa et al., 2005). Chez le cochon, il a été montré que les CSM favorisaient la cicatrisation en sécrétant du collagène de type I et en se différenciant en fibroblastes au niveau de la zone lésé lors d'une lésion induite par une irradiation (Ai et al., 2002).

Au cours de nos études antérieures concernant d'autres organes dans le même modèle préclinique de greffe de CSM humaines (CSMh) chez la souris NOD/SCID (Bensidhoum et al., 2004 ; Semont et al., 2006 ; François et al., 2006) et dans un modèle de primate non-humain irradié greffé avec des CSM de la même espèce (Chapel et al., 2003), l'administration des CSM n'a pas entraîné d'effet secondaire notable.

L'ensemble de ces connaissances suggère que les CSM semblent être des bons candidats pour le traitement thérapeutique des altérations tissulaires et qu'il existe des éléments convergents pour utiliser les CSM humaines dans la régénération de la peau. 


\section{Matériel et méthode}

Un total de 40 souris NOD/SCID de 8 semaines a été utilisé pour cette étude. Avant exposition aux rayonnements ionisants les souris ont été immobilisées par une anesthésie générale. Les souris ont été localement irradiées au niveau de la patte droite postérieure à une dose de $30 \mathrm{~Gy}$, le reste du corps étant à l'extérieur du champ d'irradiation. La dosimétrie physique par diodes thermoluminescentes a permis de vérifier la dose délivrée et la localisation de l'irradiation.

Les CSMh ont été isolées à partir de moelle osseuse de donneurs consentants et sains, conformément aux procédures approuvées par le comité d'éthique de l'hôpital Saint Antoine. Les CSM ont été isolées et amplifiées pendant 15 jours de culture en milieu complémenté. Avant injection un comptage est réalisé avec un colorant d'exclusion (bleu trypan) afin d'évaluer la viabilité cellulaire. Suivant le protocole décrit par Bensidhoum (Bensidhoum et al., 2004). Les CSMh ont été injectées par voie intraveineuse à une concentration de $2,5 \times 10^{6}$ cellules par souris, vingt quatre heures après irradiation. Vingt souris ont subi une injection (souris traitées) et 20 animaux ont été irradiées suivant la même configuration mais sans injection de CSM (non traités).

Afin de faciliter les observations, les pattes des souris ont été épilées après irradiation. Un score visuel adapté suivant les modèles de Douglas (Douglas et Fowler, 1976) ou de Hoeller (Hoeller et al., 2003) (RTGO/EORTC et LENT/SOMA) a été réalisé sur une période de 8 semaines post-irradiation ( 0 normal, 1 desquamation sèche, 2 desquamation humide, 3 ulcération, 4 nécrose). Dans le but d'apprécier les effets de la thérapie cellulaire sur les dommages de la peau, la largeur des lésions (en millimètre) a été également mesurée.

Les animaux ont été sacrifiés par groupe de 10 (5 traités et 5 non traités) à différents temps: 3,$4 ; 6$ et 8 semaines post-irradiation. Chaque échantillon de peau prélevée est scindé en deux parties. Une partie sert à la quantification de la présence des CSMh par amplification du gène de bêta-globine humaine par PCR quantitative. La seconde partie est fixée dans le formol et mise en paraffine pour réaliser l'étude histologique. L'analyse histologique comprend une étude de l'architecture tissulaire des peaux irradiées par une coloration HES (Hematoxyline, Eosine, Safran) et un immunomarquage de la protéine humaine bêta-2-microglobuline permettant de mettre en évidence la localisation in situ des CSMh dans la peau irradiée. Les résultats sont reportés sous forme de moyenne \pm écart standard à la moyenne (ESM). Les comparaisons entre groupes sont réalisées avec le test t. Les différences sont considérées comme significatives pour une valeur de $\mathrm{p}<0,01\left(^{*}\right)$ et $\mathrm{p}<0,005(* *)$ 


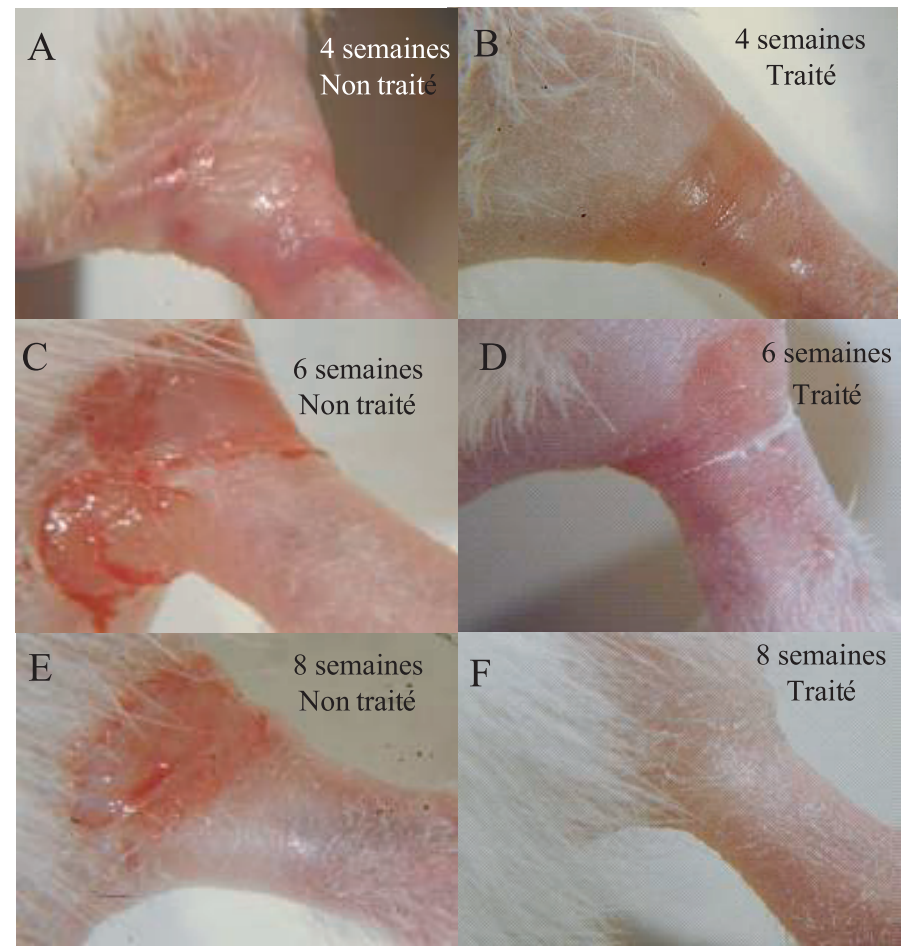

Figure 1 - Bénéfice de l'injection des CSM sur l'évolution clinique des lésions de la patte irradiée non traitée (à gauche) ou traitée (à droite). La peau des animaux traités est moins lésée et présente une cicatrisation accélérée par rapport au contrôle non traité. Quatre semaines après irradiation $(30 \mathrm{~Gy})$ les souris non-traitées présentent une atteinte sévère de la peau avec ulcération $(A)$. À des temps comparables après irradiation les animaux traités présentent une desquamation humide $(B)$. À 6 semaines $(C:$ non traité, $D:$ traité) et à 8 semaines $(E:$ non traité, F : traité) après irradiation, la cicatrisation est accélérée chez les souris traités. Grossissement initial $\times 20$.

Skin aspect of the irradiated leg of untreated (left panel) and treated (right panel) mice. The skin of hMSC injected NOD/SCID mice (treated) was less injured by irradiation and showed an accelerated wound healing when compared to the skin of controls mice (untreated). Four weeks after irradiation with a single 30 Gy dose untreated mice showed a severe injury to the skin (A) and evidenced ulceration (white arrow). At the same time post irradiation, only moist desquamation was observed in treated mice $(B)$. At six (C untreated and $D$ treated mice) and eight ( $E$ untreated and $F$ treated mice) weeks after irradiation level of healing of mouse skin were also related to $\mathrm{hMSC}$ injection. Original magnification $\times 20$.

\section{Résultats}

L'observation macroscopique des lésions 4 semaines post-irradiation (Fig. 1) et les analyses histologiques correspondantes (Fig. 2) montrent que les lésions cutanées 


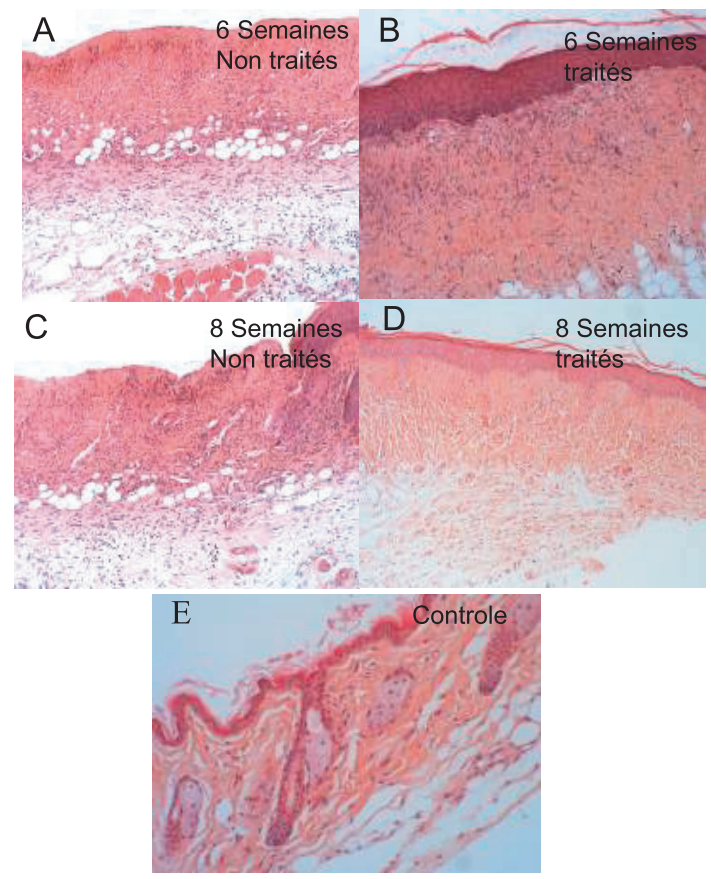

Figure 2-Bénéfice de l'injection des CSM sur l'évolution clinique des lésions de la patte. L'examen histologique, 6 et 8 semaines après irradiation des animaux non-traités (à gauche) et traités (à droite), met en évidence une ré-épithalialisation plus rapide pour les animaux traités. Quatre semaines après irradiation, les animaux non-traités présentent une ulcération $(A)$ alors que les animaux traités présentent une cicatrisation partielle avec un derme fibreux, et une inflammation du derme et de l'hypoderme $(B)$. 6 semaines après irradiation, la peau irradiée des animaux non-traités présente des zones nécrotiques avec un derme fibreux, et une inflammation du derme et de l'hypoderme $(C)$. Une cicatrisation partielle est observée après 8 semaines $(E)$ alors que les animaux traités présentent une re-épithélialisation avec un derme inflammatoire et fibreux après 6 semaines $(D)$. La cicatrisation est complète avec une fibrose modérée à 8 semaines. Coloration hématoxyline et éosine, grossissement initial $\times 50$.

The irradiated skin of treated mice showed an accelerated re-epithelialization when compared to the skin of untreated mice. Histological examination of irradiated skin samples from untreated or treated mice 6 and 8 weeks after irradiation. At 6 weeks skin wounds of untreated mice (A) exhibited ulcerated areas with a fibrous and inflamed dermis (white arrow) and hypodermis (black arrow). The inflammatory reaction was observed even deeper than the hypodermis. Treated mice (B) showed areas of reepithelialization (white arrow) with a fibrous and inflammatory dermis (black arrow) at 6 weeks. Partial healing of untreated mouse skin (C) was observed at 8 weeks. Ulcerated areas and epithelial tongue migration (black arrow) were observed. The dermis was inflammatory and fibrous (white arrow). The hypodermis exhibited signs of inflammation and fibrosis. An almost complete healing of the treated mouse (D) skin with an important re-epithelialization was observed at 8 weeks. The dermis was moderately inflammatory and fibrous (white arrow). The hypodermis was partly fibrous (black arrow). Figure $E$ represent unirradiated skin. Tissues were stained with haematoxylin and eosin, original magnification $\times 50$. 
des animaux traités par les CSM n'évoluent pas jusqu'à l'ulcération (Fig. 1B) contrairement aux animaux non traités (Fig. 1A). À six et huit semaines postirradiation l'observation de la zone irradiée (Fig. 1) et les analyses histologiques (Fig. 2) montrent une cicatrisation de la lésion cutanée radio induite chez les souris traitées (Figs. 1D, 1F et 2B, 2D) contrairement aux animaux non traités (Figs. 1C, $1 \mathrm{E}$, et $2 \mathrm{~A}, 2 \mathrm{C})$. Quatre semaines après exposition aux rayonnements ionisants (RI) la taille de la lésion cutanée est également significativement moins importante chez les souris traitées (Fig. 3B) de 2 à 4 semaines ( $p<0,005)$, la différence demeure significative jusqu'à 8 semaines $(\mathrm{p}<0,01)$. Les scores des atteintes (Fig. 3A) et de la cicatrisation (Fig. $3 \mathrm{C}$ ) réalisés sur les 4 groupes de 10 souris permettent de confirmer de manière significative que, chez les animaux traités, les lésions de la peau sont plus modérées ( $\mathrm{p}<0,005$ de 2 à 5 semaines, $\mathrm{p}<0,01$ jusqu'à 8 semaines) et la cicatrisation plus rapide que pour les souris non traitée $(\mathrm{p}<0,01$ de 3 à 4 semaines et $\mathrm{p}<0,005$ jusqu'à 8 semaines). L'extension de la patte est également plus importante (Figs. 4A et 5C) et la perte de masse musculaire est plus faible (Figs. 4B et 5C) en comparaison des souris non greffées (Figs. 4A, 4B et 5B). L'ensemble de ces résultats met en évidence une récupération fonctionnelle de la patte irradiée dépassant le simple niveau de la peau mais également des tissus sous-jacents.

Les résultats des marquages immunohistochimiques ont mis en évidence la présence de cellules humaines dans la peau altérée par une exposition aigue aux rayonnements ionisants (Fig. 6B) La faible implantation des CSMh dans la peau de la patte irradiée a été confirmée par PCR quantitative (Fig. 6C) suggérant une action stimulatrice de la reprise fonctionnelle et structurale des tissus irradiés ne nécessitant que très peu de CSMh exogènes présentes au sein du tissu altérée.

\section{Discussion}

Le présent travail concerne les atteintes cutanées radio induites. Nous avons mis au point une configuration d'irradiation induisant une atteinte sévère localisée aux RI, où les souris NOD/SCID sont uniquement irradiées au niveau de la patte droite postérieure. Après avoir effectué une gamme de dose, nous avons retenu pour cette étude une dose de 30 Gy permettant à la fois d'induire un syndrome cutané et de suivre les animaux pendant 8 semaines. Les animaux ont été greffés avec des CSMh et comparés au contrôle non greffé. La présence de CSMh dans la peau de souris 20 jours après injection est en accord avec les résultats précédemment publiés suggérant que l'incorporation des cellules transplantés dans la structure de la peau altérée par les RI pourrait être masquée par une forte présence de cellules inflammatoires aux temps précoces (Borue et al., 2004 ; Savill et Sherratt, 2003). Nos résultats antérieurs sur un modèle de primate non humain soumis à une irradiation mixte gamma-neutron ont permis précédemment de montrer que les CSM 

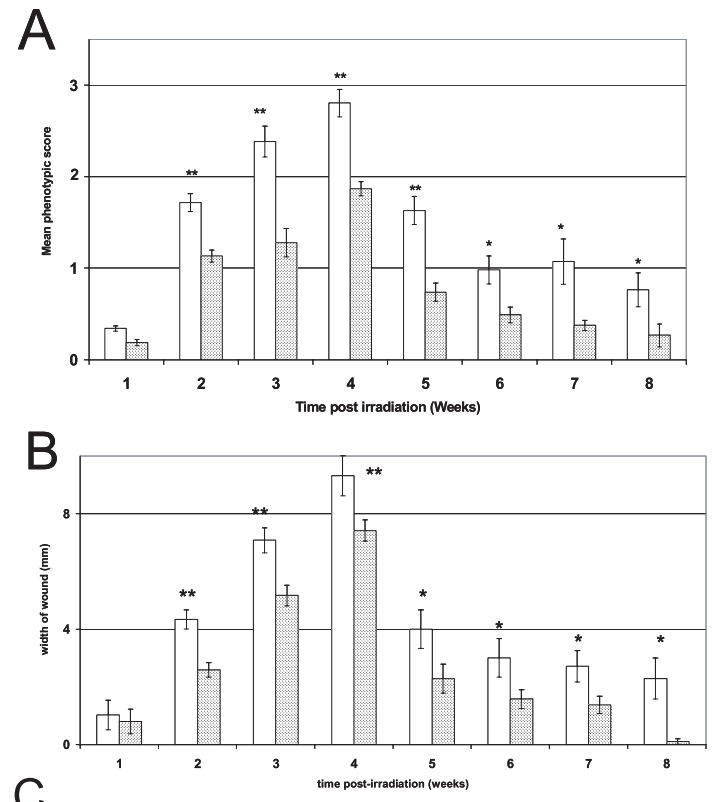

C

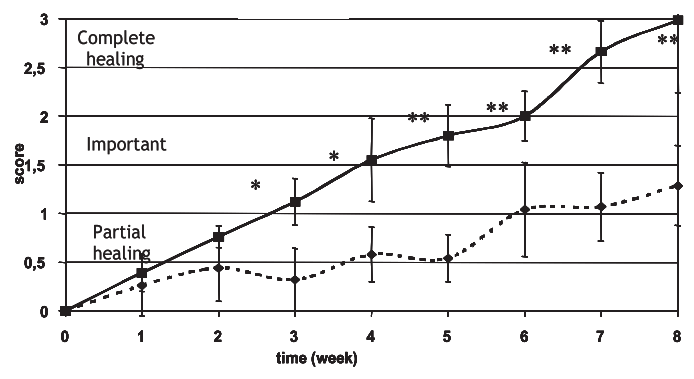

Figure 3 - Bénéfice de l'injection des CSM sur les lésions de la patte. (A) Score phénotypique pour les animaux traités (histogrammes grisés) et non traités (histogrammes blancs). (B) Diamètre des lésions ( $\mathrm{mm}$ ) après irradiation chez les souris traités ou non. (C) Score de cicatrisation (0 - pas de cicatrisation, 1 - cicatrisation partielle, 2 - cicatrisation importante, 3 - cicatrisation complète) au cours du temps pour les animaux traités (ligne continue) et non traités (pointillés). Les résultats représentent la moyenne et l'écart à la moyenne $(n=20$ par groupe $; * p<0,01$; $* * p<0,005)$.

Scores of treated mice were significantly improved when compared to untreated mouse scores. (A) Phenotypic score for the effects of irradiation on skin of treated (shaded histograms) and untreated (white histograms) mice. (B) Wound size measurement (mm) after irradiation. The size of skin wounds of the treated (bold line) mice was measured and compared to untreated (hatched line) mice. (C) Phenotypic score of healing for the effects of irradiation on skin of treated (bold line) and untreated (hatched line) mice skin (0 - normal, 1 - partial healing, 2 important healing, 3 - complete healing). Results represent the mean $\pm S E M, n=20$ for each group $(* * p<0.005$, *p $<0.01$, NS not significant). 


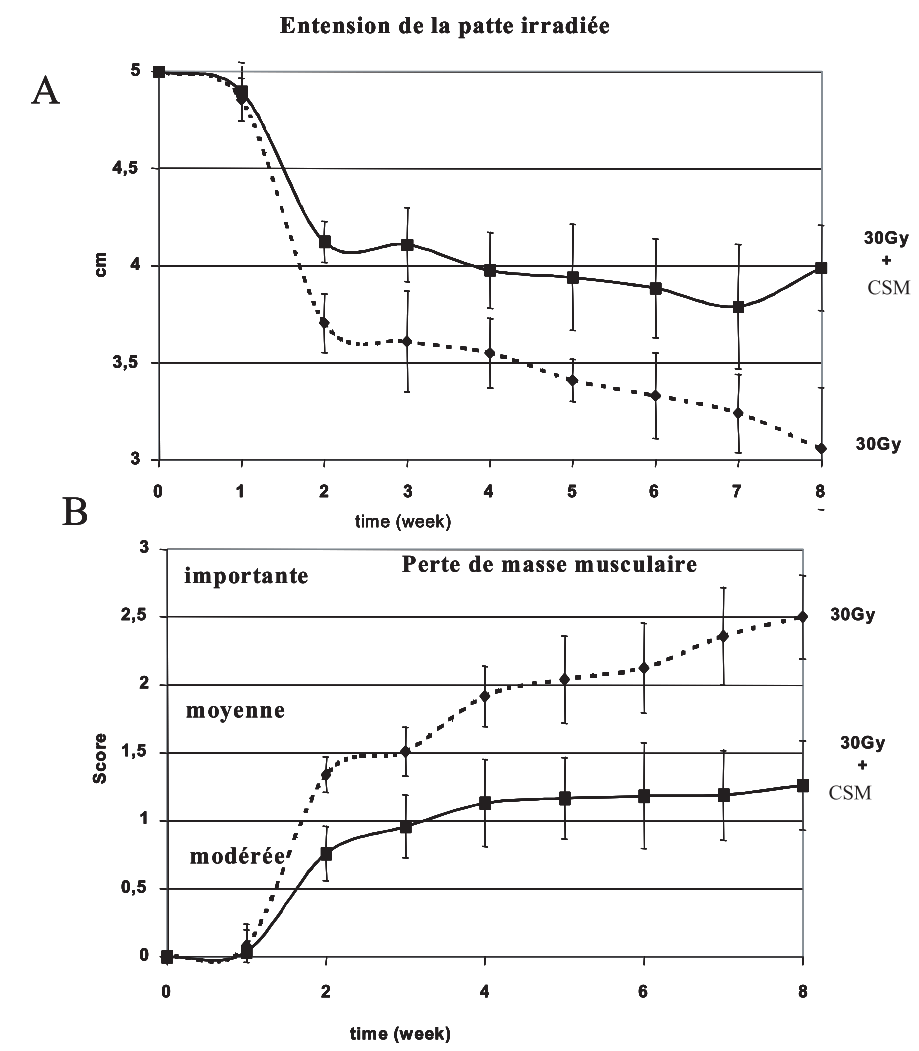

Figure 4 -Amélioration fonctionnelle du membre irradié au cours du temps pour les animaux traités (ligne continue) et non traités (ligne pointillée). (A) Extension de la patte en cm, (B) score pour la masse musculaire. Les résultats représentent la moyenne et l'écart à la moyenne $(n=20$ par groupe; *p<0,01;**p<0,005).

Scores of treated mice were significantly improved when compared to untreated mouse scores. (A) Size measurement (in cm) of the extension of irradiated posterior leg after irradiation. The size of extended leg of the treated (bold line) mice was measured and compared to untreated (hatched line) mice. (B) Phenotypic score of the loss of muscular volume for the effects of irradiation on muscle of treated (bold line) and untreated (hatched line) mice skin (0 normal, 1 moderate, 2 average, 3 important). Results represent the mean $\pm S E M, n=20$ for each group $(* * p<0.005, * p<0.01$, NS not significant).

se localisaient préférentiellement dans les tissus en régénérations. Nous avons également pu mettre en évidence que la migration des CSM exogènes dépend de la configuration, de la dose et du temps post-irradiation sur un modèle murin NOD/SCID. Nous avons constaté que les CSM humaines migraient préférentiellement dans les zones surexposées (Chapel et al., 2003 ; François et al., 2006). Ces 


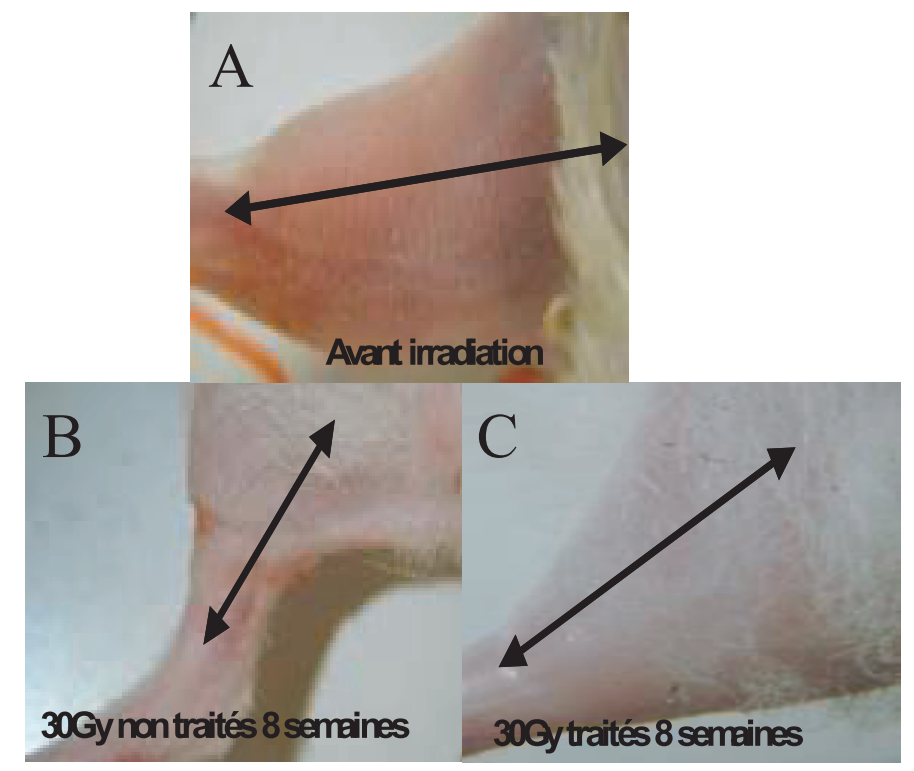

Figure 5 -Amélioration fonctionnelle du membre irradié après 8 semaines. Aspect de la patte avant irradiation (A) et irradiée non traitée (B) ou traitée $(C)$. L'extension de la patte (flèches noires) est plus importante et la fonte musculaire est plus modérée par rapport au contrôle non traité. Grossissement initial $\times 20$.

The size and the volume of muscle of extended leg of the treated $(C)$ mice was measured and compared to untreated $(B)$ mice 8 weeks after irradiation, $(A)$ non-irradiated leg. The leg extension of treated mice (black arrow) is important and the loss of muscle volume is moderated compared to untreated mice . Original magnification $\times 20$.

cellules souches adultes pourraient participer au maintien de l'homéostasie tissulaire après irradiation.

Nos résultats mettent en évidence que chez les animaux traités par les CSM humaines en comparaison aux animaux non traités :

- les lésions sont moins sévères,

- la cicatrisation est plus rapide,

- la récupération fonctionnelle de la patte irradiée est plus rapide.

D'autres études expérimentales menées parallèlement au sein de notre laboratoire ont montré que la greffe de CSM humaines diminue l'apoptose radio induite dans l'intestin après une irradiation aigue de l'abdomen (Semont et al., 2006), empêche l'apparition d'une cytotoxicité hépatique induite par une irradiation corps entiers (François et al., 2006) Les CSM transplantées dans un organisme irradié semblent participer à régénération tissulaire et au maintien de l'homéostasie 



Figure 6 - L'implantation de CSM présence de cellules humaines dans la peau de souris a été mise en évidence en immunohistologie par un anticorps anti-beta 2 microglobuline humaine (coloration brune). Figure A contrôle négatif. Figure B montrant une cellule humaine (flèche blanche) parmi les kératinocytes de souris. Grossissement initial $\times 50$.

Human cells are present in the irradiated skin of treated mice. Presence of human cells in mouse skin was assessed by immunohistology and PCR analysis. Figure A represents negative control (rabbit IgG). Figure B show an isolated human cell (white arrow) in contact with keratinocytes at 3 weeks post transplant. Detection of anti-beta-2-microglobulin primary antibody was carried out with a biotinylated secondary antibody, stained with alkaline phosphatase (fast red) followed by counterstaining with hemalyn. Original magnification $\times 50$. (C) Implantation of human MSC in irradiated skin samples was followed by quantitative PCR analysis from 3 to 8 weeks after irradiation (number of animals: 3 weeks $n=5,4$ weeks $n=5$, 6 weeks $n=5,8$ weeks $n=5$, black lines represent the means). Human beta-GLOBIN gene was found up to 8 weeks in the skin of the irradiated mice. 
tissulaire après irradiation. D'après Caplan (Caplan et Dennis, 2006), les facteurs sécrétés par les CSM inhiberaient la fibrose et l'apoptose, augmenteraient l'angiogenèse et stimuleraient la mitose et la différenciation des cellules endogènes favorisant la régénération tissulaire. Ces effets trophiques seraient distincts des mécanismes de différenciation des CSM en cellules spécifiques du tissu. L'ensemble de ces travaux suggère que la thérapie cellulaire par les CSM peut être une stratégie innovante et efficace pour la régénération des tissus lésés suite à une irradiation accidentelle. L'ensemble de ces résultats s'inscrit dans une démarche d'étude préclinique de l'utilisation de la thérapie cellulaire pour la gestion médicale des lésions précoces d'une irradiation accidentelle sévère localisée au niveau de la peau.

Actuellement, pour les patients irradiés localement à de fortes doses et en cas d'ulcération massive, l'exérèse des tissus nécrosés doit être envisagée permettant une greffe de peau sur un territoire sain. En France, cette technique de recouvrement des brûlures est pratiquée au Centre de traitement des brûlés (Dr H. Carsin). Cette approche pose des problèmes de qualité de cicatrisation et de récidive sur la zone greffée. L'ensemble de nos résultats a permis de proposer un protocole thérapeutique par injection de CSM afin de traiter un patient ayant subi des brûlures cutanées radiologiques à la main et au dos lors d'un accident au Chili. Le patient a été traité avec succès par une thérapie innovante de greffe de peau et d'injections autologues de CSM à l'Hôpital d'instruction des Armées de Percy en collaboration avec le Centre de transfusion sanguine des Armées (J.J. Lataillade).

\section{RÉFÉRENCES}

Ai G. et al. (2002) The experimental study of bone marrow mesenchymal stem cells on the repair of skin wound combined with local radiation injury, Zhonghua Yi Xиe Za Zhi 82, 1632-1636.

Archambeau J.O., Pezner R., Wasserman T. (1995) Pathophysiology of irradiated skin and breast, Int. J. Radiat. Oncol. Biol. Phys. 31, 1171-1185.

Badavias E., Abedi M., Butmarc J., Falanga V., Quesenberry P. (2003) Participation of bone marrow derived cells in cutaneous wound healing, J. Cell. Physiol. 196, 245-250.

Bensidhoum M., Chapel A., Francois S., Mazurier C., Fouillard L., Bouchet S., Bertho J.M., Gourmelon P., Aigueperse J., Charbord P., Gorin N.C., Thierry D., Lopez M. (2004) Homing of in vitro expanded Stro-1- or Stro-1+ human mesenchymal stem cells into the NOD/SCID mouse and their role in supporting human CD34 cell engraftment, Blood 103, 3313-3319.

Borue X., Lee S., Grove J., Herzog E., Harris R., Diflo T., Glusac E., Hyman K., Theise N., Krause D. (2004) Bone marrow-derived cells contribute to epithelial engraftment during wound healing, Am. J. Pathol. 165, 1767-1772.

Brittan M., Braun K.M., Reynolds L.E., Conti F.J., Reynolds A.R., Poulsom R., Alison M.R., Wright N.A., Hodivala-Dilke K.M. (2005) Bone marrow cells engraft within the epidermis and proliferate in vivo with no evidence of cell fusion, J. Pathol. 205, 1-13.

Caplan A.I., Dennis J.E. (2006) Mesenchymal Stem Cells as trophic mediators, J. Cell. Biochem. 98, 1076-1084. 
Chapel A., Bertho J.M., Bensidhoum M., Fouillard L., Young R.G., Frick J., Demarquay C., Cuvelier F., Mathieu E., Trompier F., Dudoignon N., Germain C., Mazurier C., Aigueperse J., Borneman J., Gorin N.Cl., Gourmelon P., Thierry D. (2003) Mesenchymal stem cells home to injured tissues when co-infused with haematopoetic cells to treat a radiation-induced multi-organ failure syndrome, J. Gen. Med. 5, 1028-1038.

Douglas B.G., Fowler J.F. (1976) The effect of multiple small doses of X rays on skin reactions in the mouse and a basic interpretation, Radiat. Res. 66, 401-426.

Fathke C., Wilson L., Hutter J., Kapoor V., Smith A., Hocking A., Isik F. (2004) Contribution of bone marrow-derived cells to skin: collagen deposition and wound repair, Stem. Cells 22, 812-822.

François S., Bensidhoum M., Mouiseddine M., Mazurier C., Allenet B., Semont A., Frick J., Saché A., Bouchet S., Thierry D., Gourmelon P., Gorin N., Chapel A. (2006) Local irradiation induces not only homing of human Mesenchymal Stem Cells (hMSC) at exposed sites but promotes their widespread engraftment to multiple organs: a study of their quantitative distribution following irradiation damages, Stem. Cells 24, 1020-1029.

Hoeller U., Tribius S., Kuhlmey A., Grader K., Fehlauer F., Alberti W. (2003) Increasing the rate of late toxicity by changing the score? A comparison of RTGO/EORTC and LENT/SOMA scores, Int. J. Radiat. Oncol. 55, 1013-1018.

Hopewell J.W. (1990) The skin: its structure and response to ionizing radiation, Int. J. Radiat. Biol. 57, 751-773.

Kataoka K., Medina R.J., Kageyama T., Miyazaki M., Yoshino T., Makino T., Huh N.H. (2003) Participation of adult mouse bone marrow cells in reconstitution of skin, Am. J. Patol. 163, 1227-1231.

Koc O.N., Lazarus H.M. (2001) Mesenchymal stem cells: heading into the clinic, Bone Marrow Transplant. 27, 235-239.

Koc O.N., Day J., Nieder M., Gerson S.L., Lazarus H.M., Krivit W. (2002) Allogeneic mesenchymal stem cell infusion for treatment of metachromatic leukodystrophy (MLD) and Hurler syndrome (MPS-IH), Bone Marrow Transplant. 30, 215-222.

Le Blanc K., Gotherstrom C., Ringden O., Hassan M., McMahon R., Horwitz E., Anneren G., Axelsson O., Nunn J., Ewald U., Norden-Lindeberg S., Jansson M., Dalton A., Astrom E., Westgren M. (2005) Fetal mesenchymal stem-cell engraftment in bone after in utero transplantation in a patient with severe osteogenesis imperfecta, Transplantation 79, 1607-1614.

Lefaix J.L., Daburon F. (1998) Diagnosis of acute localized irradiation lesions: review of the French experimental experience, Health Phys. 75, 375-384.

Mansilla E. et al. (2005) Human mesenchymal stem cells are tolerized by mice and improve skin and spinal cord injuries, Transplantation Proceedings 37, 292-294.

Mori L. et al. (2005) Fibrocytes contribute to the myofibroblast population in wounded skin and originate from the bone marrow, Exp. Cell. Res. 304, 81-90

Nakagawa H., Akita S., Fukui M., Fujii T., Akino K. (2005) Human mesenchymal stem cells successfully improve skin-substitute wound healing, Brit. J. Dermatol. 153, 29-36.

Peter R.U. (2002) Management of skin injuries in radiation accidents: the cutaneous radiation syndrome, in: The Medical Basis of Radiation Accidents Preparedness: The Clinical Care of Victims, R.C. Ricks, M.E. Berger, F.M. O'Hara (Eds). Parthenon, New York, pp. 225-229.

Peter R.U. (2005) Cutaneous radiation syndrome in multiorgan failure, Br. J. Radiol. Suppl. 27, 180184.

Savill N.J., Sherratt J.A. (2003) Control of epidermal stem cell clusters by Notch-mediated lateral induction, Dev. Biol. 258, 141-153.

Semont A., Francois S., Mouiseddine M., Francois A., Saché A., Frick J., Thierry D., Chapel A. (2006, in press) Mesenchymal Stem Cells Increase Self-Renewal of Small Intestinal Epithelium and Accelerate Structural Recovery After Radiation Injury, in: Tissue Engineering, a special edition 
S. FRANÇOIS et al.

of the Advances in Experimental Medicine and Biology, F. Fisher (Ed). Springer, New York, Vol. 585, pp. 19-30.

Yamaguchi Y., Kubo T., Murakami T., Takahashi M., Hakamata Y., Kobayashi E., Yoshida S., Hosokawa K., Yoshikawa K., Itami S. (2005) Bone marrow cells differentiate into wound myofibroblasts and accelerate the healing of wounds with ewposed bones when combined with an occlusive dressing, Br. J. Dermatol. 152, 616-622.

Weimin D., Qin H., Lianming L., Changhong L., Wei G., Zhigang Z., Shengguo Y., Hongye D., Ferid M., Zhao R.C.H. (2005) Engrafted Bone Marrow-Derived Flk- $1^{+}$Mesenchymal Stem Cells Regenerate Skin, Tissue Engineering 11, 110-119. 